\title{
Ischemic Stroke-Stenting versus Surgery for Carotid Disease
}

\author{
Swaroop Pawar, MD, MPH${ }^{1}$ and Steven R Messé, MD² \\ 1. Fellow, Vascular Neurology; 2. Assistant Professor of Neurology, Department of Neurology, Hospital of the University of Pennsylvania
}

\begin{abstract}
Extracranial internal carotid artery stenosis is one of the most common and best studied causes of stroke. Revascularization with carotid endarterectomy (CEA) has been shown to be beneficial for patients with severe stenosis associated with stroke or transient ischemic attack (TIA) and for many patients with moderate stenosis associated with stroke or TIA. CEA has also been shown to be beneficial for patients with asymptomatic severe stenosis if they have a reasonable expected lifespan and surgical risk, but the benefit is greater for men compared with women. Carotid angioplasty and stenting (CAS) has become a viable alternative procedure for carotid revascularization with less risk of major bleeding complications and cranial nerve injury. Randomized studies of CEA versus CAS have found that the endovascular approach is associated with a lower risk of myocardial infarction but a higher risk of peri-procedural stroke which has a greater impact on long-term quality of life. Thus, recommending CEA or CAS must be based upon individual patient characteristics and their preferences, but at this point it appears that most patients should still be receiving CEA if an intervention is required.
\end{abstract}

\section{Keywords}

Stroke prevention, carotid stenosis, carotid endarterectomy, carotid angioplasty and stenting, carotid revascularisation

Disclosure: The authors have no conflicts of interest to declare.

Received: November 18, 2011 Accepted: December 22, 2011 Citation: US Neurology, 2011;7(2):120-5 DOl: 10.17925/USN.2011.07.02.120

Correspondence: Steven R Messé, MD, Hospital of the University of Pennsylvania, 3400 Spruce Street, 3 West Gates Building, Philadelphia, PA 19104.

E: messe@mail.med.upenn.edu

The purpose of this article is twofold: first, to review the studies comparing carotid endarterectomy (CEA) with medical treatment to help decide who should undergo revascularization; and secondly, to review studies comparing carotid angioplasty and stenting (CAS) versus CEA to see how they should be revascularized.

Extracranial internal carotid artery stenosis is a leading cause of ischemic strokes and transient ischemic attacks (TIAS). It is estimated that extracranial atherosclerotic carotid disease is responsible for 15-20\% of strokes and treatments for extracranial internal carotid artery stenosis are among the best-studied interventions for preventing stroke. ${ }^{1-3}$ Several groundbreaking studies in the 1990s confirmed the benefit of surgical revascularization for most patients with hemodynamically significant carotid stenosis. At this point, CEA is considered to be the gold standard treatment for symptomatic carotid stenosis and many patients with asymptomatic carotid stenosis also undergo revascularization. ${ }^{4-10}$ With advances in endovascular techniques, CAS has evolved into a viable alternative to CEA and considerable interest has been shown in determining whether endovascular treatment is comparable to surgery for the treatment of carotid stenosis.

Determining whether a carotid stenosis has been symptomatic or asymptomatic is essential to deciding whether an individual patient would benefit from a revascularization procedure, as well as the urgency required to undertake such an intervention. Carotid artery stenosis is considered symptomatic if the patient has experienced focal neurologic symptoms related to ischemia in the ipsilateral retina causing monocular blindness, or in the ipsilateral cerebral hemisphere, potentially causing contralateral hemiparesis, hemianesthesia, a visual field cut, and neglect in the non-dominant hemisphere, or aphasia in the dominant hemisphere.

\section{Carotid Endarterectomy in Symptomatic Carotid Stenosis}

In the 1990s, two large randomized controlled trials, namely the North American symptomatic carotid endarterectomy trial (NASCET) $)^{4,6}$ and the European carotid surgery trial (ECST), ${ }^{5,7}$ established that patients with symptomatic carotid stenosis benefit from CEA.

NASCET was a randomized prospective multicenter trial carried out to assess the efficacy of CEA versus medical treatment in patients with symptomatic carotid atherosclerotic disease. The study enrolled 659 patients who had a hemispheric or retinal TIA or a non-disabling stroke within the 120 days before entry. The result showed a significant benefit of CEA in patients with 70-99\% symptomatic stenosis. The two-year ipsilateral stroke risk was $26 \%$ in the medically treated patients versus $9 \%$ in the surgical group $(p<0.001)$. The absolute risk reduction (ARR) was $17.0 \%$ and the number needed to treat (NNT) was found to be six at two years. In patients with 50-69\% symptomatic stenosis, the benefit was more modest; the five-year rate of ipsilateral stroke was $15.7 \%$ in patients treated with surgery and $22.2 \%$ in patients who received 
medical treatment (ARR $6.5 \%$, NNT 15.4, p=0.045). Finally, with $<50 \%$ symptomatic stenosis, there was no significant difference, with a five-year rate of ipsilateral stroke of $14.9 \%$ in the CEA group and $18.7 \%$ in the medical therapy group $(p=0.16){ }^{4,6}$ Subset analysis found that patients who were aged 75 and older benefited more from CEA than younger patients. ${ }^{11}$ Post hoc analyses further revealed gender differences in the 50-69\% group, with a statistical benefit from CEA seen only in men but not in women.

ECST was a multicenter randomized controlled trial that enrolled 3,024 patients who had at least one transient or mild symptomatic ischemic vascular event within the previous six months due to ipsilateral carotid artery stenosis. ${ }^{5}$ ECST initially used a different approach from NASCET to the measurement of the degree of carotid stenosis but they subsequently re-analyzed the angiography data to be consistent with NASCET (see Figure 1 for NASCET criteria). Surgery reduced the five-year risk of any stroke or surgical death by $5.7 \%$ (95\% confidence interval [Cl] 0-11.6) in patients with 50-69\% stenosis by NASCET criteria $(n=646, p=0.05)$ and by $21.2 \%$ (95 \% Cl 12.9-29.4) in patients with 70-99\% stenosis by NASCET criteria without 'near-occlusion' ( $n=429, p<0.0001)$. Thus results of the ECST and NASCET were very consistent. ${ }^{12}$

\section{Pooled Analysis and Subset Analysis of Carotid Endarterectomy Trials}

Analysis of the pooled data from the NASCET, ECST, and the Veterans Affair 309 study (a smaller randomized trial involving 189 patients with symptomatic carotid stenosis) confirmed the efficacy of CEA in patients with symptomatic carotid disease. ${ }^{13}$ The analysis showed that surgery increased the five-year risk of ipsilateral ischemic stroke in patients with less than $30 \%$ carotid stenosis, had no effect in patients with 30-49\% carotid stenosis, and was of marginal benefit in those with 50-69\% carotid stenosis. However, surgery was highly beneficial in patients with $\geq 70 \%$ carotid stenosis but not near-occlusion. Importantly, surgical morbidity and mortality exceeding $6 \%$ in symptomatic stenosis could negate the benefit gained from CEA. 14,15

The combined data allowed for more precise subgroup analyses. For timing of the procedure, it was seen that CEA was most beneficial if carried out within the first two weeks after a non-disabling stroke or TIA. ${ }^{16}$ In general, men benefit from CEA more than women with symptomatic carotid stenosis revascularization; however, CEA is clearly beneficial for women with 70-99 \% symptomatic carotid stenosis. ${ }^{17}$ Some symptomatic subgroups appeared to derive more benefit from CEA and these include patients aged 75 years or more, patients with ulcerated plaques, and patients with recent TIAs within two weeks of randomization. ${ }^{16}$ CEA is also likely to be beneficial for patients who have symptomatic ipsilateral carotid stenosis and co-existing severe contralateral carotid stenosis or occlusion, in spite of the increased risk compared with medical treatment alone. ${ }^{18}$ The interactions between all of these factors are complicated, but it is worth noting that time to CEA has the greatest impact on the potential benefit for women, such that it is reasonable to consider revascularizing women with 50-69\% stenosis, if this is carried out within two weeks of the first ischemic event. ${ }^{19}$

There was no clear benefit of the procedure in patients with total or near-total occlusion of the symptomatic ipsilateral internal carotid

\section{Figure 1: Measurement of an Internal Carotid Artery Stenosis Using the North American Symptomatic Carotid Endarterectomy Trial Criteria}

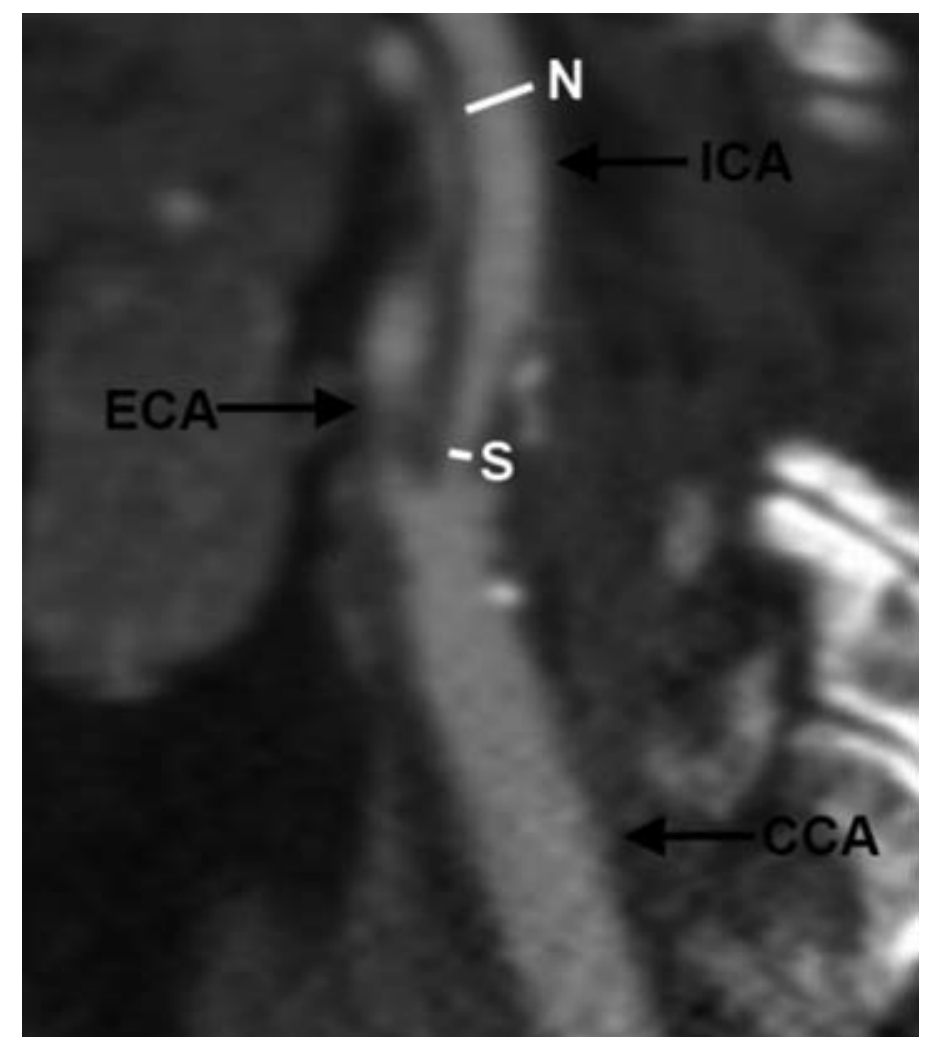

The diameter of the artery in the projection that displays the greatest degree of stenosis $(S)$ and at a normal segment distal to the stenosis $(\mathrm{N})$. The percentage of stenosis $=(1-\mathrm{S} / \mathrm{N}) \times 100$ $C C A=$ common carotid artery; $E C A=$ external carotid artery; ICA = internal carotid artery.

artery, and it is unknown whether patients with ipsilateral stroke with disabling deficits or severe comorbidities due to a medical or surgical condition would benefit or not. ${ }^{20}$

\section{Carotid Angioplasty and Stenting in Symptomatic Carotid Disease}

Based upon the previously discussed studies, CEA is considered the gold standard therapy for patients with symptomatic severe carotid stenosis. CAS is an attractive alternative as it is less invasive and associated with less cranial nerve injury and fewer bleeding complications. Within the past five years, considerable high-level data have become available as multiple randomized studies that have compared CAS to CEA have been completed.

The Stent-protected angioplasty versus carotid endarterectomy (SPACE) trial was an international, multicenter, randomized controlled European study designed to test the non-inferiority of CAS to CEA for the treatment of severe symptomatic carotid stenosis. One thousand two hundred patients with symptomatic carotid artery stenosis were randomly assigned within 180 days of TIA or moderate stroke (modified Rankin scale score of $\leq 3)$ to carotid artery stenting $(n=605)$ or CEA $(n=595) .{ }^{21}$ The primary endpoint (rate of ipsilateral ischemic stroke or death occurring within 30 days of the procedure) was $6.8 \%$ in CAS and $6.3 \%$ in CEA (absolute difference of $0.51 \%, 90 \% \mathrm{Cl}-1.89-2.91)$, which was greater than the 
Table 1: Symptomatic Carotid Disease-What Do the Guidelines Recommend?

\section{Symptomatic Carotid Artery Stenosis}

1. For patients with recent TIA or ischemic stroke within the past six months and ipsilateral severe (70-99\%) carotid artery stenosis, CEA is recommended if the peri-operative morbidity and mortality risk is estimated to be $<6 \%$

2. For patients with recent TIA or ischemic stroke and ipsilateral moderate (50-69\%) carotid stenosis, CEA is recommended depending on patient-specific factors, such as age, sex, and comorbidities, if the peri-operative morbidity and mortality risk is estimated to be $<6 \%$

3. When the degree of stenosis is $<50 \%$, there is no indication for carotid revascularization by either CEA or CAS

4. When CEA is indicated for patients with TIA or stroke, surgery within two weeks is reasonable, rather than delaying surgery, if there are no contraindications to early revascularization

5. CAS is indicated as an alternative to CEA for symptomatic (Class I; level of patients at average or low risk of complications associated evidence B) with endovascular intervention when the diameter of the lumen of the internal carotid artery is reduced by $>70 \%$ by non-invasive imaging or $>50 \%$ by catheter angiography

6. Among patients with symptomatic severe stenosis (>70 \%) in whom the stenosis is difficult to access surgically, medical conditions are present that greatly increase the risk for surgery, or when other specific circumstances exist, such as radiation-induced stenosis or restenosis after CEA, CAS may be considered

7. CAS in the above setting is reasonable when performed by operators with established peri-procedural morbidity and mortality rates of 4-6\%, similar to those observed in trials of CEA and CAS

8. For patients with symptomatic extracranial carotid occlusion, EC/IC bypass surgery is not routinely recommended

9. Optimal medical therapy, which should include antiplatelet therapy, statin therapy, and risk factor modification, is recommended for all patients with carotid artery stenosis and a TIA or stroke as outlined elsewhere in this guideline

The revised American Heart Association/American Stroke Association (AHA/ASA) guidelines published in 2011 for the prevention of stroke make the above recommendations for the management of symptomatic carotid stenosis. CAS = carotid angioplasty and stenting; $C E A=$ carotid endarterectomy; $E C / I C=$ extracranial/intracranial; $T I A=$ transient ischemic attack. Source: Furie et al., 2011. ${ }^{41}$

predefined threshold. Thus, the study failed to prove the non-inferiority of CAS compared with CEA for the peri-procedural complication rate. In a post hoc analysis, older age in the CAS group (but not the CEA group) was significantly associated with an increased risk of ipsilateral stroke or death.22 After two years follow-up, there was no statistically significant difference between CAS and CEA in the composite endpoint of any peri-procedural stroke or death and ipsilateral ischemic stroke up to two years after the procedure in both intention-to-treat (9.5 versus $8.8 \%$ ) and per-protocol (9.4 versus $7.8 \%$ ) analyses. The incidence of recurrent carotid stenosis $\geq 70 \%$ at two years, as defined by ultrasound, was significantly higher after carotid artery stenting in both analyses
(10.7 versus $4.6 \%$ by intention-to-treat). However, it cannot be excluded that the degree of in-stent stenosis is slightly overestimated by conventional ultrasound criteria. ${ }^{23}$

The Endarterectomy versus angioplasty in patients with symptomatic severe carotid stenosis (EVA-3S) was a French multicenter clinical trial that randomized 527 patients to endarterectomy $(n=262)$ or CAS $(n=265)$ to prove non-inferiority of CAS to CEA in low-risk patients with symptomatic carotid stenosis of $\geq 60 \%$. The 30-day incidence of any stroke or death, the composite primary outcome measure, was significantly higher with CAS than with CEA (9.6 versus $3.9 \%$, relative risk [RR] 2.5, $95 \% \mathrm{Cl} 1.2-5.1$ ) and the incidence of disabling stroke or death was 1.5 versus $3.4 \%$ for the CEA and CAS groups, respectively. The trial was stopped prematurely due to an excess number of deaths in the CAS group. ${ }^{24}$ At four years of follow-up, it was found that the cumulative probability of peri-procedural stroke or death and non-procedural ipsilateral stroke was higher with stenting than with endarterectomy (11.1 versus $6.2 \%$, hazard ratio [HR] $1.97,95 \% \mathrm{Cl} 1.06-3.67, \mathrm{p}=0.03)$. There were more major local complications after stenting and more systemic complications (mainly pulmonary) after endarterectomy, but the differences were not significant. Cranial nerve injury was more common after endarterectomy than after stenting. ${ }^{24}$

The International carotid stenting study (ICSS) was a multicenter European trial, in which 1,713 patients (age $>40$ years) with recently symptomatic carotid artery stenosis were randomly assigned to receive carotid artery stenting $(n=855)$ or CEA $(n=858) .{ }^{25}$ The 30-day risk of stroke, death, or myocardial infarction (MI) was significantly higher after CAS than after CEA (7.4 versus $4.0 \%$, RR 1.8, $95 \%$ Cl 1.2-2.8, $p=0.003$ ). The 120-day risk of stroke, death, or MI was still higher in the stenting cohort ( 8.5 versus $5.2 \% ; p=0.006$ ). Moreover, in a subset of 231 patients in the ICSS who had brain magnetic resonance imaging (MRI), the proportion of patients with new ischemic brain lesions on diffusion-weighted MRI at a median of one day after treatment was significantly higher in the stenting group than in the endarterectomy group (50 versus $17 \%$, odds ratio 5.2 , $95 \% \mathrm{Cl} 2.8-9.8, \mathrm{p}<0.0001){ }^{26}$

It is important to note that, relative to CEA, CAS is a less mature procedure and, as with any intervention, experience and improvements in techniques and devices have an impact on the potential risk and efficacy. Many proponents of CAS have argued that EVA-3S, SPACE, and ICSS did not insure that the interventionists had adequate experience with CAS prior to enrolling patients in the study. That said, analyses of the experience of interventionists in these studies did not show a relationship with peri-procedural events. ${ }^{27-30}$

Most recently, the Carotid revascularization endarterectomy versus stenting trial (CREST) was published. ${ }^{31}$ CREST was a North American randomized multicenter trial comparing CAS with CEA in both symptomatic and asymptomatic patients. The primary endpoint was the occurrence of stroke, death, or $\mathrm{Ml}$ during the peri-procedural period and ipsilateral stroke up to four years. About half of the patients enrolled had an asymptomatic $>60 \%$ stenosis and half were symptomatic with $>50 \%$ stenosis. The CREST study attempted to address the issue of inexperienced interventionists by enforcing a credentialing lead-in period of up to 20 CAS procedures prior to enrolling patients in the study. Overall, there was no significant difference in the rates of the primary endpoint 
between CAS and CEA (7.2 versus $6.8 \%$, HR 1.11, $95 \% \mathrm{Cl} 0.81-1.51$, $\mathrm{p}<0.51$ ) during long-term follow-up (median 2.5 years). An interaction with age and treatment was detected $(p<0.02)$. Outcomes were slightly better after CAS for patients aged $<70$ years and better after CEA for patients aged $>70$ years. The proportion of patients developing stroke within 30 days of the procedure was significantly higher in the CAS than the CEA group (4.1 versus $2.3 \%$, HR 1.8, $95 \% \mathrm{Cl} 1.1-2.8$ ); on the other hand, the frequency of $\mathrm{MI}$ within 30 days of the procedure was significantly lower in the CAS group (1.1 versus $2.3 \%$, HR 0.5, $95 \% \mathrm{Cl} 0.3-0.9$ ). However, at one-year follow-up the quality of life was significantly diminished for patients who developed stroke compared with those with MI. ${ }^{31}$

For the subgroup of patients with symptomatic carotid disease, the peri-procedural rate of stroke and death was significantly higher for those assigned to stenting compared with endarterectomy (6.0 versus $3.2 \%$, HR 1.89, $95 \% \mathrm{Cl} 1.1-3.2) .{ }^{32}$ In addition, CREST found that the difference in peri-procedural complications between CEA and CAS was accentuated in women. Peri-procedural events occurred in 35 (4.3\%) of 807 men assigned to carotid artery stenting compared with 40 (4.9\%) of 823 assigned to CEA (HR 0.90, $95 \% \mathrm{Cl} 0.57-1.41)$ and 31 (6.8\%) of 455 women assigned to carotid artery stenting compared with 16 (3.8\%) of 417 assigned to CEA (HR 1.84, $95 \%$ Cl 1.01-3.37, interaction $\mathrm{p}=0.064)$. $^{33}$

\section{Asymptomatic Carotid Stenosis}

Whether to recommend revascularization for an asymptomatic carotid stenosis is a question that has persistently troubled neurologists, due to the fact that prior studies have found variable benefit from revascularization, based upon a number of different patient characteristics. In addition, advances in medical therapies have raised doubts on whether the original CEA studies would show similar results if they were performed now.

\section{Carotid Endarterectomy in Asymptomatic Carotid Stenosis}

The Asymptomatic carotid atherosclerosis study (ACAS) was a prospective randomized multicenter trial that randomized 1,662 patients with asymptomatic carotid stenosis of $>60 \%$ to CEA and medical management. ${ }^{8}$ After a median follow-up of 2.7 years, the aggregate five-year risk of ipsilateral stroke, any peri-operative stroke, or death was estimated to be 5 versus $11 \%$ for an RR reduction of 0.53 (95 \% Cl 0.22-0.72) favoring CEA. However, for surgery to be beneficial, the rate of peri-operative death and other serious complications had to be less than $3 \%$, and the expected patient survival had to be at least five years. The study showed higher incidence of peri-operative complications in women compared with men (3.6 versus $1.7 \%$ ), and men had an ARR of $8 \%$ compared with $1.4 \%$ in women, with women receiving no statistically significant benefit from revascularization.

The Asymptomatic carotid surgery trial (ACST) was a subsequent randomized multicenter trial that enrolled 3,120 patients with $\geq 60 \%$ asymptomatic carotid stenosis but no recent neurological symptoms (stroke or TIA) between immediate intervention (CEA) or indefinitely deferred CEA (until there was an associated stroke or TIA). Of 1,560 patients allocated to immediate treatment, half had CEA by one month and $88 \%$ by one year, and of the deferred group only $4 \%$ per year underwent CEA. ${ }^{9}$ The CEA group had a peri-operative risk of stroke or
Table 2: Asymptomatic Carotid Disease-What Do the Guidelines Recommend?

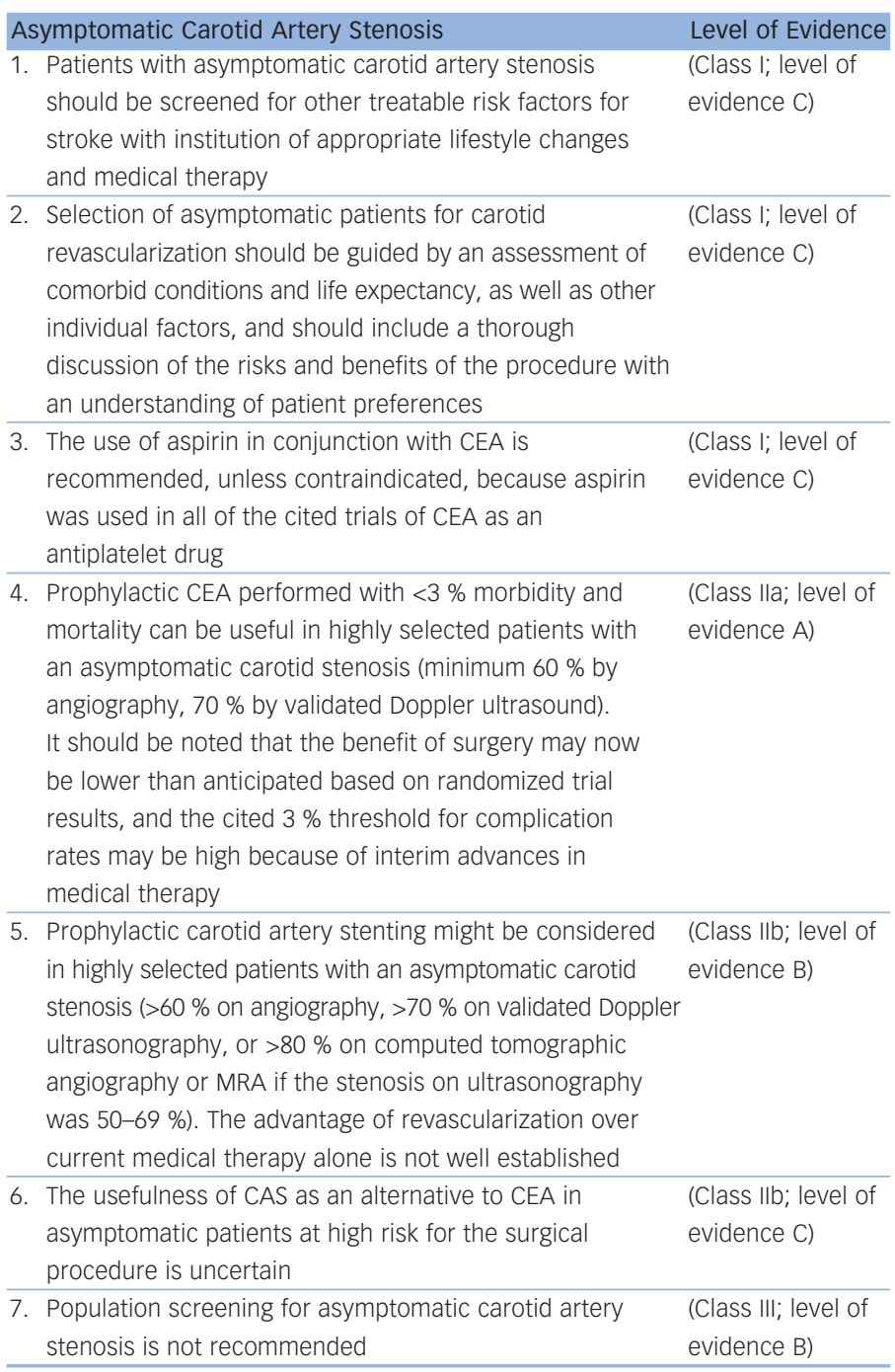

The revised American Heart Association/American Stroke Association (AHA/ASA) guidelines published in 2011 for the primary prevention of stroke make the above recommendations for the management of asymptomatic carotid disease. CAS = carotid angioplasty and stenting; $C E A=$ carotid endarterectomy; $M R A=$ magnetic resonance angiography. Source: Goldstein et al., 2011.42

death of $3.1 \%$ within 30 days of surgery; however, the net five-year risk for all strokes or peri-operative death in the immediate CEA group was reduced by nearly half compared with the CEA deferral group (6.4 versus $11.8 \%, 95 \% \mathrm{Cl} 2.96-7.75)$, results that are similar to the ACAS study.

The ARR for preventing non-peri-operative stroke over five years was greater for men than for women $(8.2 \%, 95 \% \mathrm{Cl} 5.64-10.78$, versus $4.08 \%$, $95 \% \mathrm{Cl}$ 0.74-7.41), although the benefit was statistically significant for both. CEA was shown to benefit patients $<75$ years of age, but there was no statistical benefit in patients who were older. ACST re-emphasized that, when selecting asymptomatic patients for carotid revascularization, age, sex, life expectancy and the cited $3 \%$ complication rate must all be taken into account. Finally, it is important to note that patients randomized to medical therapy in both ACAS and ACST were undertreated in terms of modern interventions such as statins and aggressive blood pressure goals. 
More recent prospective cohorts of medically treated patients with asymptomatic carotid stenosis have reported much lower stroke rates than these trials. A review of data collected from 11 studies showed that the average annual rates of ipsilateral or any other ischemic stroke in asymptomatic severe carotid stenosis fell significantly in the last three decades with medical management alone. ${ }^{34}$ It remains unknown whether modern aggressive medical management would be better than, or equivalent to, revascularization in asymptomatic carotid stenosis, but it is an area which is gaining attention. Finally, it may be possible to identify patients at higher risk of stroke who then may be most likely to benefit from revascularization. Based upon the ACST study, progression of carotid atherosclerosis on serial Doppler ultrasound studies is associated with increased risk of first stroke. ${ }^{35}$ Furthermore, though not routinely available at every hospital, prolonged microembolic signal detection with transcranial Doppler (TCD) ultrasound has been validated in a multicenter study to accurately stratify high- and low-risk patients with asymptomatic carotid stenosis. ${ }^{36}$

In summary, CEA can be recommended in men who have a life expectancy of at least five years with asymptomatic carotid stenosis of 60-99\%, provided the peri-operative risk of stroke and death is $<3 \%$. In women, carotid revascularization can be considered, particularly if they are younger and have a low expected peri-procedural risk.

\section{Carotid Angioplasty and Stenting in Asymptomatic Carotid Stenosis- Stenting and Angioplasty with Protection in Patients at High Risk for Endarterectomy}

Besides CREST, as discussed earlier, another study that looked into the utility of CAS in asymptomatic patients was the Stenting and angioplasty with protection in patients at high risk for endarterectomy (SAPPHIRE) trial. SAPPHIRE randomized 334 high-surgical-risk patients to receive CAS with emboli protection device or endarterectomy to test the hypothesis that CAS was not inferior to endarterectomy. These patients had symptomatic carotid stenosis of $\geq 50 \%$ or asymptomatic carotid stenosis of $\geq 80 \%$; however, more than $70 \%$ of patients had asymptomatic carotid disease. ${ }^{37,38}$ Patients were considered high risk for revascularization based upon age $>80$, pulmonary or cardiac disease, prior neck surgery, or radiation. The primary endpoint of the cumulative incidence of a major cardiovascular event at one year, which included a composite of peri-procedural death, stroke, or Ml (within 30 days after the procedure), and/or death or ipsilateral stroke between 31 days and one year, was $12.2 \%$ for CAS compared with $20.1 \%$ for CEA (absolute difference $7.9 \%, 95 \% \mathrm{Cl}-0.7-16.4 \%$ ).

In the analysis of symptomatic patients with carotid stenosis (30\% in the CAS and $28 \%$ in the endarterectomy group), the cumulative incidences of the primary endpoint at 30 days, and also one year, in both groups were non-significantly different ( 2.2 versus $9.3 \%, p=0.18$ at 30 days, and 16.8 versus $16.5 \%, p=0.95$ at one year). For patients with asymptomatic carotid stenosis (70 \% in the CAS and $72 \%$ in the endarterectomy group), the cumulative incidence of the primary endpoint in the peri-procedural period was 5.4 versus $10.2 \%(p=0.20)$ in the CAS and CEA groups, respectively. SAPPHIRE hinted that CAS is not inferior to CEA in high-risk patients, namely with contralateral carotid occlusion, neck irradiation, prior neck surgery, severe cardiac/pulmonary disease, recurrent stenosis post-CEA, and age more than 80 years. ${ }^{39}$

In patients $\geq 80$ years old, a meta-analysis of 41 studies of either CEA or CAS showed that the stroke rate was significantly higher for CAS compared with CEA (7.0 versus $1.9 \%)$; the relative risks of death or $\mathrm{Ml}$ at 30 days were fairly similar. ${ }^{40}$

As mentioned above, half of the patients included in the CREST study had an asymptomatic carotid revascularization. For these patients, the stroke and death rates were $2.5 \pm 0.6 \%$ for CAS and $1.4 \pm 0.5 \%$ for CEA (HR 1.88, $95 \% \mathrm{Cl} 0.79-4.42, \mathrm{p}=0.15)$.

\section{Conclusions}

The decision to recommend carotid revascularization, by what method, and in what timeframe, needs to be done on an individual basis depending on specific patient characteristics and the availability of surgeons and interventionists with a high volume of procedures and a track record with an acceptable complication rate. The accompanying tables provide the current recommendations from the American Heart Association regarding carotid revascularization (see Tables 1 and 2). Clearly, the vast majority of patients with symptomatic carotid stenosis of 70-99\% will benefit from revascularization, which should be carried out as soon as possible, assuming that the first event was not a major stroke. In this setting, it appears that CEA is more beneficial compared with CAS when the lesion is surgically accessible. CAS may be a reasonable option in this setting if the lesion is surgically inaccessible, if there is restenosis post-CEA, radiation-induced stenosis, or underlying comorbidities increasing surgical risk, assuming that the expected peri-procedural risk remains less than $6 \%$.

CEA does offer benefit, compared with medical treatment, for asymptomatic carotid stenosis ranging between 60 and $99 \%$, provided the life expectancy goal of five years is met and the combined peri-operative risk of stroke or death associated with the procedure is less than $3 \%$, though the benefit is greater in men compared with women. Based on the most recent data from CREST, CAS may be a reasonable choice in patients with an asymptomatic stenosis, particularly for younger men.

Lastly, it is important to bear in mind that the trials comparing medical therapy with CEA were designed and implemented over the last three decades. During this time, there have been significant changes in medical management for the prevention of stroke. With the advent of newer antiplatelet agents, more powerful statins with more aggressive lipid goals, use of non-beta-blocker blood pressure medications with more aggressive goals, decreased prevalence of smoking, and increased awareness of diet and exercise, there is ample evidence that the risk of stroke from an otherwise asymptomatic carotid stenosis is less than the risk seen in prior CEA studies. In some patients with asymptomatic stenosis, aggressive medical management alone rather than CEA could be offered with serial non-invasive imaging studies to ensure that the stenosis is not progressing. Microembolic signal detection with TCD may also help to risk-stratify asymptomatic patients. 
1. Sacco RL, Kargman DE, Gu Q, Zamanillo MC, Race-ethnicity and determinants of intracranial atherosclerotic cerebral infarction. The Northern Manhattan Stroke Study, Stroke, 1995;26(1):14-20.

2. Wolff $T$, Guirguis-Blake J, Miller $T$, et al., Screening for carotid artery stenosis: an update of the evidence for the U.S. Preventive Services Task Force, Ann Intern Med, 2007:147(12):860-70.

3. Wityk RJ, Lehman D, Klag M, et al., Race and sex differences in the distribution of cerebral atherosclerosis, Stroke, 1996:27(11):1974-80.

4. Beneficial effect of carotid endarterectomy in symptomatic patients with high-grade carotid stenosis. North American Symptomatic Carotid Endarterectomy Trial Collaborators, N Engl J Med, 1991;325(7):445-53.

5. MRC European Carotid Surgery Trial: interim results for symptomatic patients with severe $(70-99 \%)$ or with mild $(0-29 \%)$ carotid stenosis. European Carotid Surgery Trialists Collaborative Group, Lancet, 1991;337(8752):1235-43.

6. Barnett HJ, Taylor DW, Eliasziw M, et al., Benefit of carotid endarterectomy in patients with symptomatic moderate or severe stenosis. North American Symptomatic Carotid Endarterectomy Trial Collaborators, N Engl J Med, 1998;339(20):1415-25.

7. Randomised trial of endarterectomy for recently symptomatic carotid stenosis: final results of the MRC European Carotid Surgery Trial (ECST), Lancet, 1998;351(9113):1379-87.

8. Endarterectomy for asymptomatic carotid artery stenosis. Executive Committee for the Asymptomatic Carotid Atherosclerosis Study, JAMA, 1995:273(18):1421-8.

9. Halliday A, Mansfield A, Marro J, et al., Prevention of disabling and fatal strokes by successful carotid endarterectomy in patients without recent neurological symptoms: randomised controlled trial, Lancet, 2004;363(9420):1491-502.

10. Halliday A, Harrison M, Hayter E, et al., 10-year stroke prevention after successful carotid endarterectomy for asymptomatic stenosis (ACST-1): a multicentre randomised trial, Lancet, 2010;376(9746):1074-84

11. Alamowitch S, Eliasziw M, Algra A, et al., Risk, causes, and prevention of ischaemic stroke in elderly patients with symptomatic internal-carotid-artery stenosis, Lancet, 2001;357(9263):1154-60

12. Rothwell PM, Gutnikov $S A$, Warlow $C P$, Reanalysis of the fina results of the European Carotid Surgery Trial, Stroke, 2003;34(2):514-23

13. Rothwell PM, Eliasziw M, Gutnikov SA, et al., Analysis of pooled data from the randomised controlled trials of endarterectomy for symptomatic carotid stenosis, Lancet, 2003:361(9352):107-16.

14. Gorelick PB, Carotid endarterectomy : where do we draw the line?, Stroke, 1999;30(9):1745-50.

15. Goldstein LB, Moore WS, Robertson JT, Chaturvedi S, Complication rates for carotid endarterectomy. A call to action, Stroke, 1997;28(5):889-90.
16. Rothwell PM, Eliasziw M Gutnikov SA et al. Endarterectomy for symptomatic carotid stenosis in relation to clinical subgroups and timing of surgery, Lancet, 2004;363(9413):915-24.

17. Kernan WN, Viscoli CM, Brass LM, et al., The stroke prognosis instrument II (SPI-II) : A clinical prediction instrument for patients with transient ischemia and nondisabling ischemic stroke, Stroke, 2000:31(2):456-62.

18. Gasecki AP, Eliasziw M, Ferguson GG, et al., Long-term prognosis and effect of endarterectomy in patients with symptomatic severe carotid stenosis and contralateral carotic stenosis or occlusion: results from NASCET. North American Symptomatic Carotid Endarterectomy Trial (NASCET) Group, J Neurosurg, 1995:83(5):778-82.

19. Rothwell PM, Mehta Z, Howard SC, et al., Treating individuals 3 : from subgroups to individuals: general principles and the example of carotid endarterectomy, Lancet, 2005;365(9455):256-65.

20. Brott TG, Brown RD Jr, Meyer FB, et al., Carotid revascularization for prevention of stroke: carotid endarterectomy and carotid artery stenting, Mayo Clin Proc, 2004:79(9):1197-208.

21. Ringleb PA, Allenberg J, Brückmann $\mathrm{H}$, et al., 30 day results from the SPACE trial of stent-protected angioplasty versus carotid endarterectomy in symptomatic patients: a randomised non-inferiority trial, Lancet, 2006;368(9543):1239-47.

22. Stingele R, Berger J, Alfke $K$, et al., Clinical and angiographic risk factors for stroke and death within 30 days after carotid endarterectomy and stent-protected angioplasty: a subanalysi of the SPACE study, Lancet Neurol, 2008;7(3):216-22.

23. Eckstein $\mathrm{HH}$, Ringleb $\mathrm{P}$, Allenberg JR, et al., Results of the Stent-Protected Angioplasty versus Carotid Endarterectomy (SPACE) study to treat symptomatic stenoses at 2 years: a multinational, prospective, randomised trial, Lancet Neurol 2008:7(10):893-902

24. Mas JL, Chatellier G, Beyssen B, et al., Endarterectomy versus stenting in patients with symptomatic severe carotid stenosis, N Engl J Med, 2006;355(16):1660-71.

25. Ederle J, Dobson J, Featherstone RL, et al., Carotid artery stenting compared with endarterectomy in patients with symptomatic carotid stenosis (International Carotid Stenting Study): an interim analysis of a randomised controlled trial, Lancet, 2010;375(9719):985-97.

26. Bonati $L H$, Jongen $L M$, Haller $S$, et al., New ischaemic brain lesions on MRI after stenting or endarterectomy for symptomatic carotid stenosis: a substudy of the International Carotid Stenting Study (ICSS), Lancet Neurol, 2010;9(4):353-62.

27. Roffi M, Sievert H, Gray WA, et al., Carotid artery stenting versus surgery: adequate comparisons?, Lancet Neurol, 2010;9(4):339-41; author reply 341-2.

28. Fiehler J, Bakke SJ, Clifton A, et al., Plea of the defence-critical comments on the interpretation of EVA3S, SPACE and ICSS,
Neuroradiology, 2010:52(7):601-10

29. Fiehler J, Jansen O, Berger J, et al., Differences in complication rates among the centres in the SPACE study, Neuroradiology, 2008;50(12): 1049-53

30. Macdonald S, Lee R, Williams R, Stansby G, Delphi Carotid Stenting Consensus Panel, Towards safer carotid artery stenting: a scoring system for anatomic suitability, Stroke, 2009;40(5):1698-703.

31. Brott TG, Hobson RW 2nd, Howard G, et al., Stenting versus endarterectomy for treatment of carotid-artery stenosis, N Eng/ J Med, 2010;363(1):11-23.

32. Silver FL, Mackey A, Clark WM, et al., Safety of stenting and endarterectomy by symptomatic status in the Carotid Revascularization Endarterectomy Versus Stenting Trial (CREST), Stroke, 2011;42(3):675-80.

33. Howard VJ, Lutsep HL, Mackey A, et al., Influence of sex on outcomes of stenting versus endarterectomy: a subgroup analysis of the Carotid Revascularization Endarterectomy versus Stenting Trial (CREST), Lancet Neurol, 2011;10(6):530-7.

34. Abbott AL, Medical (nonsurgical) intervention alone is now best for prevention of stroke associated with asymptomatic severe carotid stenosis: results of a systematic review and analysis, Stroke, 2009;40(10):e573-83.

35. Hirt LS, Progression Rate and Ipsilateral Neurological Events in Asymptomatic Carotid Stenosis, Stroke, 2011 [Epub ahead of print].

36. Markus HS, King A, Shipley M, et al, Asymptomatic embolisation for prediction of stroke in the Asymptomatic Carotid Emboli Study (ACES): a prospective observational study, Lancet Neurol, 2010;9(7):663-71.

37. Yadav JS, Wholey MH, Kuntz RE, et al., Protected carotid-artery stenting versus endarterectomy in high-risk patients, N Engl / Med, 2004:351(15):1493-501.

38. Yadav JS, Carotid stenting in high-risk patients: design and rationale of the SAPPHIRE trial, Cleve Clin I Med 2004;71(Suppl. 1):S45-6

39. Cambria RP, Stenting for carotid-artery stenosis, N Engl J Med, 2004;351(15):1565-7.

40. Usman AA, Tang GL, Eskandari MK, Metaanalysis of procedura stroke and death among octogenarians: carotid stenting versus carotid endarterectomy, J Am Coll Surg, 2009;208(6):1124-31.

41. Furie KL, Kasner SE, Adams RJ, et al., Guidelines for the prevention of stroke in patients with stroke or transient ischemic attack: a guideline for healthcare professionals from the American Heart Association/American Stroke Association, Stroke, 2011:42(1):227-76.

42. Goldstein LB, Bushnell CD, Adams RJ, et al., Guidelines for the primary prevention of stroke: a guideline for healthcare professionals from the American Heart Association/American Stroke Association, Stroke, 2011;42(2):517-84. 